\title{
Effects of Adiponectin on T2DM and Glucose Homeostasis: A Mendelian Randomization Study
}

This article was published in the following Dove Press journal:

Diabetes, Metabolic Syndrome and Obesity: Targets and Therapy

\author{
Zefeng Chen $\mathbb{D}^{1-5}$ \\ Yulan Bai ${ }^{1-5}$ \\ Xinyang Long ${ }^{1-5}$ \\ Qianqian Luo ${ }^{1-5}$ \\ Zheng Wen ${ }^{1-5}$ \\ Yuanfan $\mathrm{Li}^{1-5}$ \\ Shengzhu Huang ${ }^{1-5}$ \\ Yunkun Yan ${ }^{1-5}$ \\ Zengnan Mol-4,6
}

'Center for Genomic and Personalized Medicine, Guangxi Medical University, Nanning 53002I, Guangxi, People's Republic of China; ${ }^{2}$ Guangxi Key Laboratory for Genomic and Personalized Medicine, Nanning 53002I, Guangxi, People's Republic of China; ${ }^{3}$ Guangxi Collaborative Innovation Center for Genomic and Personalized Medicine, Nanning 53002I, Guangxi, People's Republic of China; ${ }^{4}$ Guangxi Key Laboratory of Colleges and Universities, Nanning 53002I, Guangxi, People's Republic of China; ${ }^{5}$ School of Public Health, Guangxi Medical University, Nanning 53002I, Guangxi, People's Republic of China; ${ }^{6}$ Institute of Urology and Nephrology, First Affiliated Hospital of Guangxi Medical University, Nanning 53002I, Guangxi, People's Republic of China

Correspondence: Zengnan Mo Center for Genomic and Personalized Medicine, Guangxi Medical University, 22 Shuangyong Road, Nanning 53002I, Guangxi, People's Republic of China Tel +8677l-5353342

Email mozengnan@gxmu.edu.cn
Purpose: The associations of adiponectin with type 2 diabetes mellitus (T2DM), glucose homeostasis (including $\beta$-cell function index (HOMA- $\beta$ ), insulin resistance (HOMA-IR), fasting insulin (FI) and fasting glucose (FG)) have reported in epidemiological studies. However, the previous observational studies are prone to biases, such as reverse causation and residual confounding factors. Herein, a Mendelian Randomization (MR) study was conducted to determine whether causal effects exist among them.

Materials and and Methods: Two-sample MR analyses and multiple sensitivity analyses were performed using the summary data from the ADIPOGen consortium, MAGIC Consortium, and a meta-analysis of GWAS with a considerable sample of T2DM $(62,892$ cases and 596,424 controls of European ancestry). We got eight valid genetic variants to predict the causal effect among adiponectin and T2DM and glucose homeostasis after excluding the probable invalid or pleiotropic variants.

Results: Adiponectin was not associated with T2DM (odds ratio $(\mathrm{OR})=1.004 ; 95 \%$ confidence interval (CI): $0.740,1.363$ ) when using MR Egger after removing the invalid SNPs, and the results were consistent when using the other four methods. Similar results existed among adiponectin and HOMA- $\beta$, HOMA-IR, FI, FG.

Conclusion: Our MR study revealed that adiponectin had no causal effect on T2DM and glucose homeostasis and that the associations among them in observational studies may be due to confounding factors.

Keywords: GWASs, adiponectin, Mendelian randomization, T2DM, glucose homeostasis

\section{Introduction}

Type 2 diabetes mellitus (T2DM), affecting no less than 400 million people all over the world, ${ }^{1}$ is a classic example of the polygenic disease. The prevalence and morbidity of T2DM are rapidly increasing worldwide. ${ }^{2,3}$ The prevalence of diabetes and prediabetes was estimated to be $11.6 \%$ and $50.1 \%$, respectively, in China, in $2013 .{ }^{4}$ The people with diabetes mellitus are evaluated to increase to 42.3 million by $20300^{5,6} \mathrm{~T} 2 \mathrm{DM}$ is also one of the critical risk factors of nonalcoholic fatty liver disease, which is considered the most common chronic liver disease globally. ${ }^{7}$ The risk of T2DM consists of two parts, genetic factors and unhealthy living, ${ }^{8}$ and the heritability estimates are moderate to high $(0.47-0.77) .{ }^{9}$ The inheritance of adiponectin is ethnically specific, whose variants at CDH13, ADIPOQ, PDE3A, RFC4, EIF4A2, and so on exhibited significant association $(P<5 \mathrm{E}-8)$ in the European ethnicity. ${ }^{10,11}$ In contrast, the variants at $\mathrm{CDH} 13, \mathrm{KNG1}-\mathrm{ADIPOQ}{ }^{12-14}(P<5 \mathrm{E}-8)$, and WDR11-FGFR2 $(P=3 \mathrm{E}$ -14) exhibited significant association in Asians. ${ }^{15}$ Besides, there also provided suggestive evidence for a locus on chromosome 12 near OR8S1-LALBA $(P=1.2 \mathrm{E}-7)$ in 
Asians. ${ }^{15}$ Adiponectin, which is an anti-inflammatory adipocytokine secreted by adipocytes, has anti-atherogenic and anti-inflammatory properties and was implicated in comprehensive biological pathways associated with insulin resistance (HOMA-IR). ${ }^{16-18}$ Inflammation has been considered the potential cause of T2DM. ${ }^{19,20}$ The destruction of the $\beta$ cell function index (HOMA- $\beta$ ) leads to an absolute lack of insulin, and impaired HOMA- $\beta$ and HOMA-IR are vital determinants of T2DM. ${ }^{21}$ Cross-sectional epidemiological studies have shown that low serum adiponectin is connected to HOMA- $\beta,^{22}$ HOMA-IR, T2DM, and obesity. ${ }^{23,24}$ In longitudinal studies, hypoadiponectinemia was able to forecast the development of HOMA-IR, T2DM, low-density lipoprotein cholesterol, and triglyceride. ${ }^{24-27}$ Increasing adiponectin is connected with a lower risk of T2DM, ${ }^{24,28}$ and inversely relates to changes in HOMA- $\beta{ }^{22}$ However, whether this correlation is causal or reverse causal or caused by confounding factors remains unknown.

Mendelian randomization (MR) utilizes genetic variants as instrumental variables (IVs) and explore the causal effect between exposure and outcome, but has to meet that the IVs associated with the exposure (IV1, $P<5 \mathrm{E}-8$ ) and the IVs do not affect the outcome through other paths other than the exposure of interest (IV2) and are not associated with confounders (IV3) (Figure 1). Any SNPs that go against these assumptions should be served as invalid IVs and removed. ${ }^{29,30}$ MR can overcome the weakness of classical epidemiological studies and provide available evidence to support or reject causal hypotheses using the existing summary data of genome-wide association studies (GWAS). ${ }^{30} \mathrm{MR}$ is not vulnerable to confounding factors because the genotype of an individual is determined at gamete formation and cannot be altered later on. Therefore, reverse causation is impossible. ${ }^{26}$ MR analyses have been conducted to explore the causal relationship between adiponectin and HOMA-IR, but the conclusion is controversial. ${ }^{23,28,31}$ Studies have shown that no causal relationship exists among adiponectin and fasting glucose (FG), fasting insulin (FI). ${ }^{31}$ Nonetheless, the result was disappointing because either the sample size was too small or multiracial issues were involved. ${ }^{23}$ Here, we will evaluate the effects of adiponectin on T2DM and glucose homeostasis utilizing the summary data of European ancestry.

\section{Materials and Methods}

\section{Study Design}

We researched the relationship between adiponectin and T2DM and four related glucose homeostasis traits

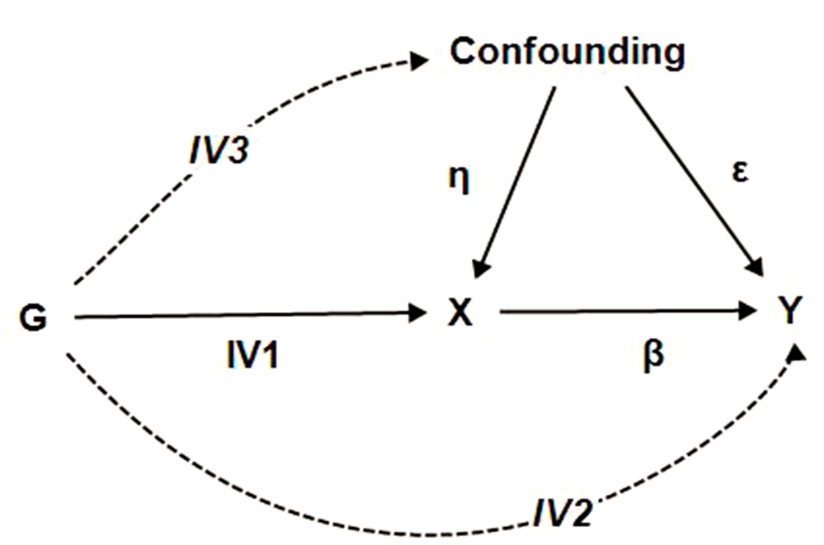

Figure I A directed acyclic graph representing the MR framework.

Notes: IVI: The $G$ associates with the $X(P<5 E-8)$; IV2: The $G$ should not straightforwardly associate with $Y$; IV3: The $G$ should not associate with any confounding factors. Abbreviations: MR, Mendelian randomization; G, multiple single nucleotide polymorphisms; $X$, exposure; $Y$, outcome; $\eta$, the correlation coefficient of confounding factors and exposure; $\varepsilon$, the correlation coefficient of confounding factors and outcome; $\beta$, the causal effect estimate of the exposure and outcome.

(HOMA- $\beta$, HOMA-IR, FI, FG) using MR. The hypotheses and study design were shown in Figure 2.

\section{Selection of IVs and Datasets Adiponectin and Glucose Homeostasis}

We identified 162 candidate variants (Table S1, https:// www.mcgill.ca/genepi/adipogen-consortium) that had genome-wide significant $(P<5 \mathrm{E}-8)$ associations with adiponectin in the ADIPOGen consortium, which conducted the meta-analysis of GWASs of adiponectin levels (European ancestry, $\mathrm{n}=39883$ ). ${ }^{10,11,16}$ When the $F$ statistic is 10 , it is equivalent to the $p$-value of 0.001 , and 30 corresponds to $5 \mathrm{E}-8 .{ }^{32}$ So our IVs are thus compelling. We removed 25 SNPs for being palindromic and 119 SNPs for being palindromic linkage disequilibrium (LD). The calculation of LD utilized the European population data of 1000 Genomes Project (CEU (Utah Residents from North and West Europe), FIN (Finnish in Finland), GBR (British in England and Scotland), IBS (Iberian population in Spain) and TSI (Toscani in Italia)) at the standard of $\mathrm{R} 2 \geq 0.1$ online (https://asia.ensembl.org/Homo_sapiens/ Tools/). The calculation of LD was conducted in the same chromosome, and the one that had the minimal $P$-value was reserved when two or more SNP is LD.

The MR study requires that IVs with known pleiotropy should be eliminated. ${ }^{33}$ In the context of adiponectin and T2DM or glucose homeostasis relationship, obesity-related traits is most likely a significant confounder. ${ }^{34-38}$ Then, the pleiotropy of 18 remaining candidate IVs (Table S2) 


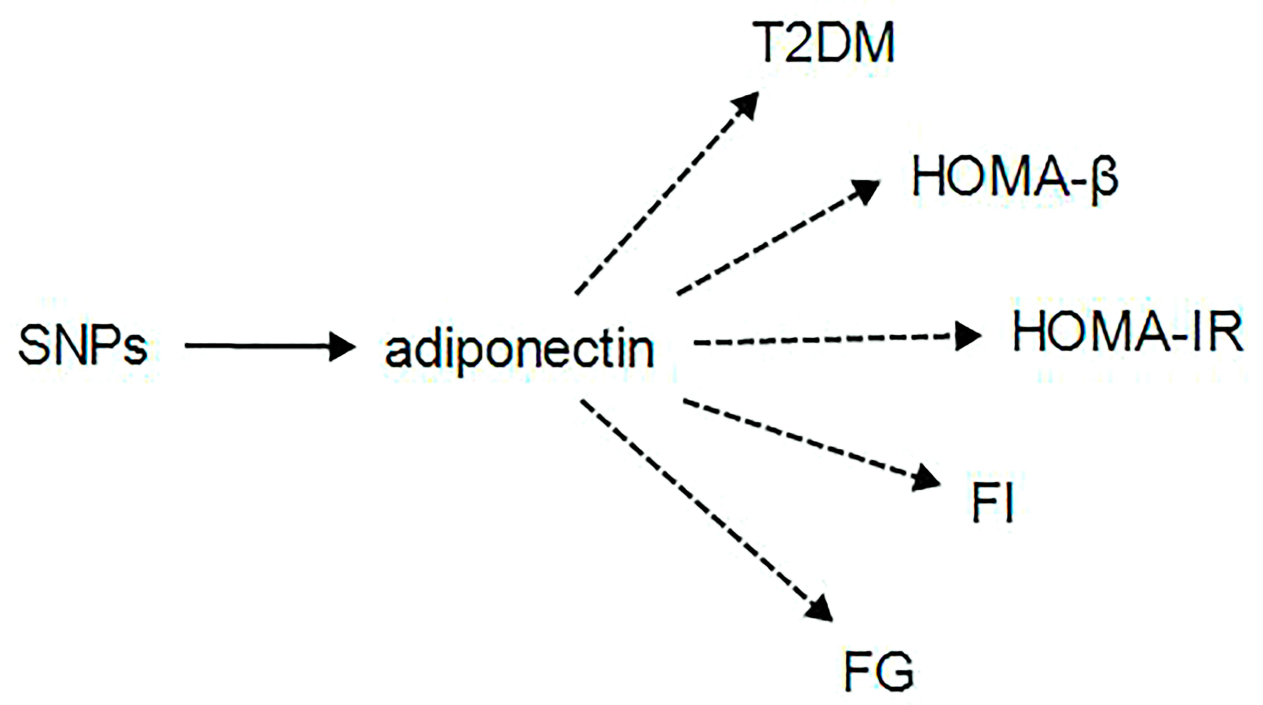

Figure 2 The unidirectional flow chart showing the relationship between adiponectin and outcomes.

Notes: To explore whether adiponectin regulated by the SNPs is the cause of T2DM and glucose homeostasis. The solid arrow represents the causal effect, and the dotted arrow may have a causal relationship.

Abbreviations: SNPs, single-nucleotide polymorphisms; T2DM, type 2 diabetes mellitus; HOMA-B, $\beta$-cell function index; HOMA-IR, insulin resistance; Fl, fasting insulin; FG, fasting glucose.

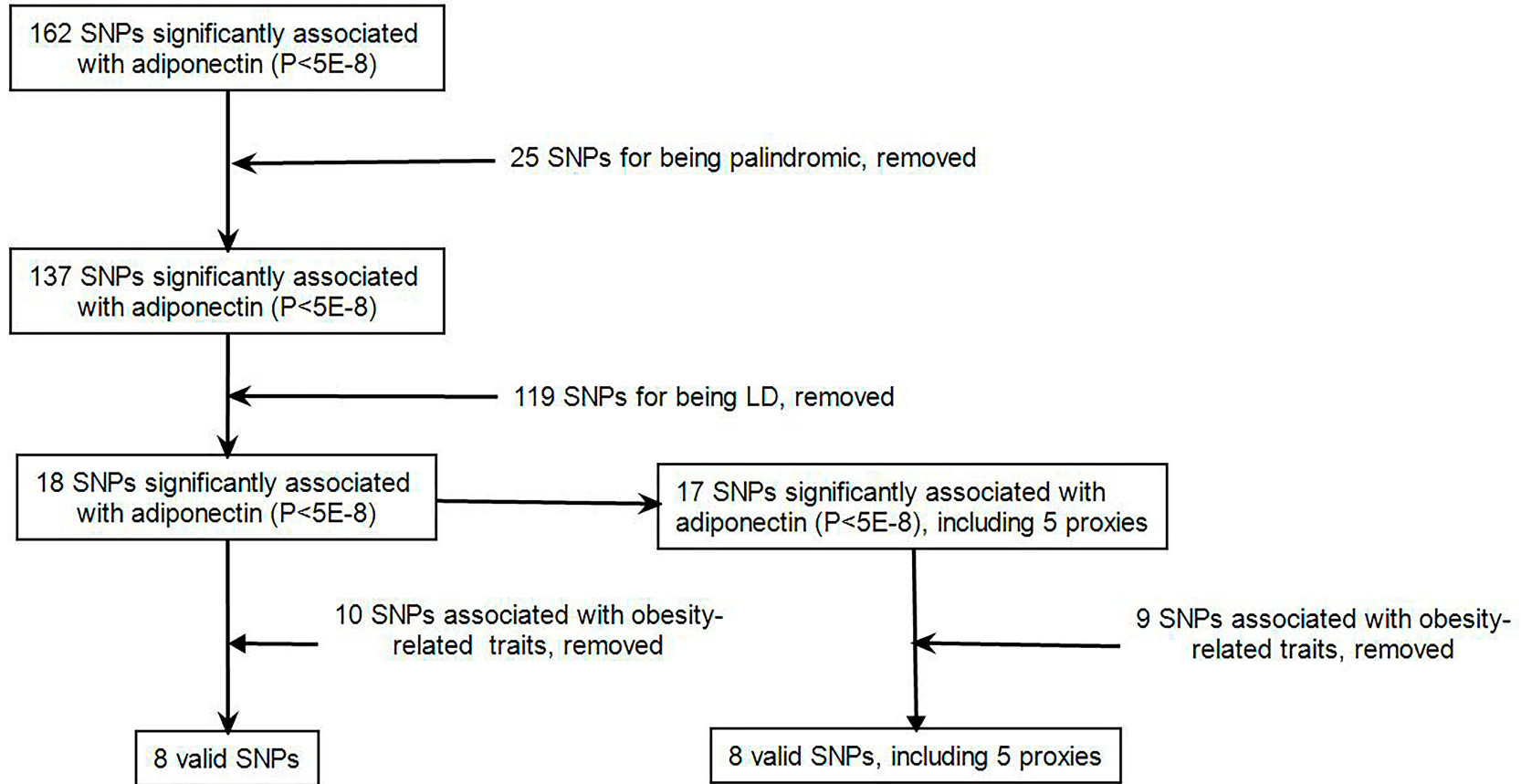

Figure 3 The flow chart of instrumental variables selection.

Notes: The 162 candidate variants that had genome-wide significant $(P<5 E-8)$ associations with adiponectin $(P<5 E-8)$ were from the ADIPOGen consortium. Abbreviations: SNPs, single-nucleotide polymorphisms; LD, linkage disequilibrium. 
Table I The Results of MR Analysis of Adiponectin and T2DM, HOMA-B, HOMA-IR, FI, FG Before and After Removed the Pleiotropic SNPs

\begin{tabular}{|c|c|c|c|c|c|c|c|c|c|}
\hline Method & $\mathbf{N}$ & P-value & SE & Beta & $\begin{array}{l}\text { Beta_ } \\
95 \% \text { LCI }\end{array}$ & $\begin{array}{l}\text { Beta_ } \\
95 \% \text { UCI }\end{array}$ & OR & $\begin{array}{l}\text { OR_ } \\
95 \% \text { LCI }\end{array}$ & $\begin{array}{l}\text { OR_- } \\
\text { 95\%UCl }\end{array}$ \\
\hline \multicolumn{10}{|c|}{ Adiponectin to T2DM } \\
\hline Egger & $17^{\mathrm{a}}$ & 0.282 & 0.230 & -0.257 & -0.708 & 0.195 & 0.774 & 0.493 & 1.215 \\
\hline IVW & $17^{\mathrm{a}}$ & 0.058 & 0.146 & 0.277 & -0.009 & 0.563 & 1.319 & 0.991 & 1.757 \\
\hline WM & $17^{\mathrm{a}}$ & 0.150 & 0.077 & 0.111 & -0.040 & 0.262 & 1.117 & 0.961 & 1.300 \\
\hline PWM & $17^{\mathrm{a}}$ & 0.151 & 0.077 & 0.110 & -0.040 & 0.261 & 1.117 & 0.960 & 1.298 \\
\hline MBE & $17^{\mathrm{a}}$ & 0.557 & 0.062 & 0.037 & -0.085 & 0.160 & 1.038 & 0.919 & 1.173 \\
\hline Egger & $8^{\mathrm{b}}$ & 0.978 & 0.156 & 0.004 & -0.301 & 0.310 & 1.004 & 0.740 & 1.363 \\
\hline IVW & $8^{\mathrm{b}}$ & 0.615 & 0.078 & 0.039 & -0.113 & 0.191 & 1.040 & 0.893 & 1.211 \\
\hline WM & $8^{\mathrm{b}}$ & 0.580 & 0.077 & 0.042 & -0.116 & 0.200 & 1.043 & 0.891 & 1.222 \\
\hline PWM & $8^{\mathrm{b}}$ & 0.595 & 0.080 & 0.042 & -0.108 & 0.192 & 1.043 & 0.898 & 1.212 \\
\hline MBE & $8^{\mathrm{b}}$ & 0.415 & 0.091 & 0.079 & -0.094 & 0.252 & 1.082 & 0.910 & 1.287 \\
\hline \multicolumn{10}{|c|}{ Adiponectin to HOMA- $\beta$} \\
\hline Egger & $18^{\mathrm{a}}$ & 0.745 & 0.030 & 0.010 & -0.048 & 0.068 & 1.010 & 0.953 & 1.071 \\
\hline IVW & $18^{\mathrm{a}}$ & 0.020 & 0.020 & 0.047 & 0.008 & 0.087 & 1.048 & 1.008 & 1.091 \\
\hline WM & $18^{\mathrm{a}}$ & 0.196 & 0.026 & 0.033 & -0.017 & 0.084 & $\mathrm{I} .034$ & 0.983 & 1.087 \\
\hline PWM & $18^{\mathrm{a}}$ & 0.183 & 0.025 & 0.033 & -0.016 & 0.081 & 1.033 & 0.985 & 1.085 \\
\hline MBE & $18^{\mathrm{a}}$ & 0.206 & 0.025 & 0.033 & -0.016 & 0.083 & 1.034 & 0.984 & 1.086 \\
\hline Egger & $8^{\mathrm{b}}$ & 0.360 & 0.033 & 0.032 & -0.032 & 0.096 & 1.033 & 0.969 & 1.101 \\
\hline IVW & $8^{\mathrm{b}}$ & 0.156 & 0.021 & 0.030 & -0.011 & 0.072 & 1.031 & 0.989 & 1.074 \\
\hline WM & $8^{\mathrm{b}}$ & 0.340 & 0.027 & 0.025 & -0.027 & 0.078 & 1.026 & 0.973 & $1.08 \mathrm{I}$ \\
\hline PWM & $8^{\mathrm{b}}$ & 0.301 & 0.024 & 0.025 & -0.025 & 0.076 & 1.026 & 0.975 & 1.079 \\
\hline MBE & $8^{b}$ & 0.586 & 0.028 & 0.016 & -0.044 & 0.076 & 1.016 & 0.957 & 1.079 \\
\hline \multicolumn{10}{|c|}{ Adiponectin to HOMA-IR } \\
\hline Egger & $18^{\mathrm{a}}$ & 0.803 & 0.039 & 0.010 & -0.067 & 0.087 & 1.010 & 0.935 & 1.091 \\
\hline IVW & $18^{\mathrm{a}}$ & 0.003 & 0.029 & 0.086 & 0.029 & 0.142 & 1.089 & 1.030 & 1.152 \\
\hline WM & $18^{\mathrm{a}}$ & 0.096 & 0.030 & 0.050 & -0.009 & 0.109 & $1.05 \mathrm{I}$ & 0.991 & 1.115 \\
\hline PWM & $18^{\mathrm{a}}$ & 0.111 & 0.031 & 0.050 & -0.012 & 0.112 & $1.05 \mathrm{I}$ & 0.989 & 1.118 \\
\hline MBE & $18^{\mathrm{a}}$ & 0.093 & 0.028 & 0.050 & -0.005 & 0.106 & 1.052 & 0.995 & 1.111 \\
\hline Egger & $8^{\mathrm{b}}$ & 0.360 & 0.033 & 0.032 & -0.032 & 0.096 & 1.033 & 0.969 & 1.101 \\
\hline IVW & $8^{\mathrm{b}}$ & 0.156 & 0.021 & 0.030 & -0.011 & 0.072 & 1.031 & 0.989 & 1.074 \\
\hline WM & $8^{\mathrm{b}}$ & 0.340 & 0.027 & 0.025 & -0.027 & 0.078 & 1.026 & 0.973 & $1.08 \mathrm{I}$ \\
\hline PWM & $8^{\mathrm{b}}$ & 0.301 & 0.024 & 0.025 & -0.026 & 0.076 & 1.026 & 0.975 & 1.079 \\
\hline MBE & $8^{\mathrm{b}}$ & 0.586 & 0.028 & 0.016 & -0.041 & 0.073 & 1.016 & 0.960 & 1.076 \\
\hline \multicolumn{10}{|c|}{ Adiponectin to FI } \\
\hline Egger & $18^{\mathrm{a}}$ & 0.721 & 0.039 & 0.014 & -0.062 & 0.090 & 1.014 & 0.940 & 1.095 \\
\hline IVW & $18^{\mathrm{a}}$ & 0.001 & 0.029 & 0.093 & 0.037 & 0.150 & 1.098 & 1.038 & 1.162 \\
\hline WM & $18^{\mathrm{a}}$ & 0.037 & 0.029 & 0.060 & 0.000 & 0.120 & 1.062 & 1.001 & 1.128 \\
\hline PWM & $18^{\mathrm{a}}$ & 0.039 & 0.029 & 0.060 & 0.002 & 0.118 & 1.062 & 1.002 & 1.125 \\
\hline MBE & $18^{a}$ & 0.062 & 0.029 & 0.058 & 0.000 & 0.116 & 1.060 & $1.00 \mathrm{I}$ & 1.123 \\
\hline Egger & $8^{\mathrm{b}}$ & 0.170 & 0.041 & 0.063 & -0.016 & 0.143 & 1.065 & 0.984 & 1.154 \\
\hline IVW & $8^{\mathrm{b}}$ & 0.034 & 0.026 & 0.055 & 0.004 & 0.106 & 1.057 & 1.004 & 1.112 \\
\hline WM & $8^{\mathrm{b}}$ & 0.067 & 0.031 & 0.057 & -0.005 & 0.119 & 1.059 & 0.995 & 1.127 \\
\hline PWM & $8^{b}$ & 0.064 & 0.031 & 0.057 & -0.004 & 0.118 & 1.059 & 0.996 & 1.126 \\
\hline MBE & $8^{b}$ & 0.148 & 0.035 & 0.057 & -0.015 & 0.130 & 1.059 & 0.985 & 1.139 \\
\hline
\end{tabular}


Table I (Continued).

\begin{tabular}{|c|c|c|c|c|c|c|c|c|c|}
\hline Method & $\mathbf{N}$ & P-value & SE & Beta & $\begin{array}{l}\text { Beta_ } \\
95 \% \text { LCI }\end{array}$ & $\begin{array}{l}\text { Beta_ } \\
95 \% \text { UCI }\end{array}$ & OR & $\begin{array}{l}\text { OR_ } \\
\text { 95\%LCl }\end{array}$ & $\begin{array}{l}\text { OR_ } \\
95 \% \mathrm{UCl}\end{array}$ \\
\hline \multicolumn{10}{|c|}{ Adiponectin to FG } \\
\hline Egger & $18^{\mathrm{a}}$ & 0.674 & 0.035 & -0.015 & -0.084 & 0.054 & 0.985 & 0.919 & 1.056 \\
\hline IVW & $18^{\mathrm{a}}$ & 0.249 & 0.022 & 0.026 & -0.018 & 0.069 & 1.026 & 0.982 & $1.07 \mid$ \\
\hline WM & $18^{\mathrm{a}}$ & 0.813 & 0.030 & 0.007 & -0.051 & 0.065 & 1.007 & 0.950 & 1.068 \\
\hline PWM & $18^{\mathrm{a}}$ & 0.804 & 0.028 & 0.007 & -0.049 & 0.063 & 1.007 & 0.953 & 1.065 \\
\hline MBE & $18^{\mathrm{a}}$ & 0.899 & 0.029 & 0.004 & -0.054 & 0.061 & 1.004 & 0.948 & 1.063 \\
\hline Egger & $8^{\mathrm{b}}$ & 0.820 & 0.040 & 0.010 & -0.069 & 0.089 & 1.010 & 0.933 & 1.093 \\
\hline IVW & $8^{b}$ & 0.861 & 0.025 & 0.004 & -0.045 & 0.054 & 1.004 & 0.956 & 1.056 \\
\hline WM & $8^{\mathrm{b}}$ & 0.932 & 0.030 & 0.003 & -0.057 & 0.062 & 1.003 & 0.945 & 1.064 \\
\hline PWM & $8^{\mathrm{b}}$ & 0.933 & 0.031 & 0.003 & -0.057 & 0.062 & 1.003 & 0.945 & 1.064 \\
\hline MBE & $8^{\mathrm{b}}$ & 0.976 & 0.033 & 0.001 & -0.070 & 0.072 & 1.001 & 0.932 & 1.075 \\
\hline
\end{tabular}

Note: ${ }^{a}$ The instrumental variables without removing the pleiotropic single nucleotide polymorphisms (SNPs); ${ }^{\mathrm{b}}$ The instrumental variables removed the pleiotropic SNPs. Abbreviations: T2DM, type 2 diabetes mellitus; HOMA-B, $\beta$-cell function index; HOMA-IR, insulin resistance; FI, fasting insulin; FG fasting glucose; Egger, MR Egger regression; IVW, Inverse variance weighting; WM, Weighted median; PWM, Penalized WM; MBE, Mode-based estimate; N, the numbers of instrumental variables; Beta, beta coefficient; SE, standard error; OR, odds ratio; $95 \% \mathrm{Cl}, 95 \%$ confidence interval; $\mathrm{LCl}$ and $\mathrm{UCl}$ are lower and upper $95 \%$ confidence intervals, respectively.

was analyzed online (PhenoScanner V2: http://www.phe $\underline{\text { noscanner.medschl.cam.ac.uk/; GWAS Catalog: https:// }}$ www.ebi.ac.uk/gwas/). We excluded 10 SNPs associated with obesity-related traits (Table S3) from the 18 SNPs associated with adiponectin. Lastly, eight valid SNPs were acquired (Figure 3). And the summary data of glucose homeostasis were obtained from a meta-analysis of 21 GWASs (46 186European non-diabetic patients) (https://www.magicinvestigators.org/downloads/). ${ }^{21}$

Summary data used in this study were freely accessible online and did not require ethical approval.

\section{Adiponectin and T2DM}

Summary data of T2DM cannot match the six SNPs (rs12051272, rs1870843, rs16861209, rs266743, rs76150 90, and rs998584) so that they needed proxies. Firstly, the proxy should exist between the summary data of exposure and outcome. Secondly, the proxy and the SNP that need proxy are in LD and has a larger Pearson's correlation. ${ }^{32}$ The proxies found were rs8047711, rs8046196, rs266719, rs2062632, and rs187868, respectively, except that rs998584 cannot find out proxy (Table S4). The process of valid IVs selection was shown in Figure 3. The summary data of T2DM were from the meta-analysis of GWASs by combining 3 GWAS data sets: Diabetes Genetics Replication and Meta-analysis (DIAGRAM), Genetic Epidemiology Research on Aging (GERA), and the UK Biobank (UKBB) (62892 T2DM cases and 596424 controls of European ancestry) (http://cnsgenomics.com/data.html). ${ }^{39}$ Following the formula used previously, ${ }^{18,40}$ we estimated the phenotypic variance explained by a given SNP (R2). Eight IVs approximatively had R2 of 3\% for T2DM and $4 \%$ for glucose homeostasis. ${ }^{32}$

\section{Statistical Analysis}

\section{Statistical Analyses for MR}

There were five MR methods used in two-sample MR analyses: (1) Inverse variance weighting (IVW) is to execute an IVW meta-analysis to acquire the MR estimate, and the weight is the inverse of the variance of SNP-outcome effect. ${ }^{10}$ When all genetic variants are valid IV, IVW is an effective method of analysis. However, even only one genetic variant is invalid, it will be biased. IVW estimates will remain biased even if there are an infinite number of IVs because all IVs would contribute to overall IVW estimate; ${ }^{29}$ (2) MR Egger regression (MR-Egger) relaxes the IV2 assumption of "no horizontal pleiotropy" and returns unbiased causal estimate. The IV2 is violated by all SNPs but meets that the hypothesis of the horizontal pleiotropic effects is uncorrelated with the SNP-exposure effects (ie, INSIDE assumption). ${ }^{29,30,41}$ If the pleiotropy of all IVs is associated with the same confounding, the InSIDE assumption will be violated; ${ }^{29}$ (3) Weighted median (WM), whose effect estimate would gain by weighting the contribution of each SNP using the inverse variance of its association with outcome. ${ }^{30} \mathrm{WM}$ would acquire an unbiased causal estimate when half of the SNPs are valid IVs (ie, meeting the needs of three assumptions). The type I error rate of WM is lower than that of the IVW method. WM is also complementary to 
Table 2 The Results of Cochran's Heterogeneity Test and MR Egger Intercept of Adiponectin and T2DM, HOMA-B, HOMA-IR, FI, FG Before and After Removed the Pleiotropic SNPs

\begin{tabular}{|c|c|c|c|c|c|c|c|c|}
\hline Outcome & $\mathbf{N}$ & $\begin{array}{l}\text { Cochran's } \\
\text { Heterogeneity } \\
\text { Test (IVW) - Q }\end{array}$ & $\begin{array}{l}\text { Cochran's } \\
\text { Heterogeneity Test } \\
\text { (IVW) - Q_pval }\end{array}$ & $\begin{array}{l}\text { Cochran's } \\
\text { Heterogeneity } \\
\text { Test (Egger) - Q }\end{array}$ & $\begin{array}{l}\text { Cochran's } \\
\text { Heterogeneity Test } \\
\text { (Egger) - Q_pval }\end{array}$ & $\begin{array}{l}\text { Intercept } \\
\text { (Egger) }\end{array}$ & se & p-val \\
\hline T2DM & $17^{\mathrm{a}}$ & 145.798 & $<0.001$ & 97.082 & $<0.001$ & 0.028 & 0.010 & 0.015 \\
\hline T2DM & $8^{b}$ & 13.313 & 0.065 & 13.161 & 0.041 & 0.002 & 0.009 & $0.80 \mathrm{I}$ \\
\hline HOMA- $\beta$ & $18^{a}$ & 19.953 & 0.277 & 17.028 & 0.384 & 0.003 & 0.002 & 0.117 \\
\hline HOMA- $\beta$ & $8^{b}$ & 2.603 & 0.919 & 2.595 & 0.858 & $<0.001$ & 0.003 & 0.930 \\
\hline HOMA-IR & $18^{a}$ & 25.539 & 0.083 & 18.310 & 0.306 & 0.005 & 0.002 & 0.023 \\
\hline HOMA-IR & $8^{\mathrm{b}}$ & 0.337 & $>0.999$ & 0.269 & $>0.999$ & -0.001 & 0.003 & 0.803 \\
\hline $\mathrm{FI}$ & $18^{a}$ & 27.316 & 0.054 & 19.032 & 0.267 & 0.005 & 0.002 & 0.018 \\
\hline $\mathrm{FI}$ & $8^{b}$ & 0.556 & 0.999 & 0.485 & 0.998 & -0.001 & 0.003 & 0.799 \\
\hline FG & $18^{a}$ & 10.344 & 0.889 & 8.160 & 0.944 & 0.003 & 0.002 & 0.159 \\
\hline FG & $8^{\mathrm{b}}$ & 1.734 & 0.973 & 1.707 & 0.945 & $<-0.001$ & 0.003 & 0.875 \\
\hline
\end{tabular}

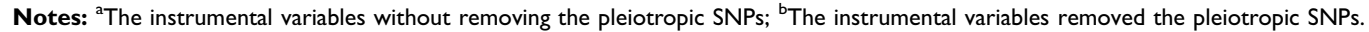

Abbreviations: T2DM, type 2 diabetes mellitus; HOMA-B, $\beta$-cell function index; HOMA-IR, insulin resistance; FI, fasting insulin; FG fasting glucose; Egger, MR Egger regression; IVW, Inverse variance weighting; N, the numbers of instrumental variables; SE, standard error; SNPs, single nucleotide polymorphisms.

MR-Egger; ${ }^{29}$ (4) Penalized weighted median (PWM), which is similar to WM. The accuracy of PWM is slightly worse than that of IVW but is marginally better in some cases. The method is consistent and more accurate than the MR-Egger method, with a standard error reduction of approximately $30 \%-50 \%{ }^{29}$ (5) Mode-based estimate (MBE) based on the similarity of IVs' causal effects, MBE clusters the SNPs into different groups and returns the causal effect estimation based on the cluster with the largest number of SNPs. If the SNPs within the maximum cluster are valid, the MBE returns an unbiased causal effect. ${ }^{41}$ We managed the MR analyses utilizing $\mathrm{R}$ (version 3.5 .3 ) with the $\mathrm{R}$ package "TwosampleMR". Two-tail $\mathrm{P}<0.05$ was considered statistically significant.

\section{Heterogeneity and Sensitivity Tests}

The heterogeneity test acts as an indicator of potential horizontal pleiotropy utilizing the Cochran's Q test, ${ }^{30,42}$ and it was considered statistically significant (indicating no horizontal pleiotropy) with a $P$-value of $>0.1$. The MR Egger intercept can be worked as the estimate of directional pleiotropy, ${ }^{43}$ and the $P$-value $<0.05$ indicates SNPs with directional pleiotropy. Furthermore, a "leave-one-out" analysis was used to assess whether the causal estimate was driven by a single SNP that may have great pleiotropy by reestimating the effect by sequentially dropping one SNP at a time. ${ }^{30}$ Singe SNP analysis was used to value the contribution of the causal effect of each SNP. We also conducted data harmonization to prevent probable bias. ${ }^{32}$

\section{Power Calculation}

We also evaluated the power for our MR analyses utilizing the statistics of the observational study using an online calculator tool mRnd (http://cnsgenomics.com/shiny/ $\underline{\mathrm{mRnd} /}$ ) (Table S5). ${ }^{44}$

\section{Results \\ MR Results}

Before removed the potential pleiotropic IVs, the four methods (IVW, WM, PWM, and MBE) showed a causal effect of adiponectin on FI ((odds ratio $(\mathrm{OR})=1.098,95 \%$ confidence interval (95\% CI): $1.038-1.162, P=0.001 ; \mathrm{OR}=1.062,95 \%$ CI: $1.001-1.128, P=0.037$; OR $=1.062$, 95\% CI: $1.002-$ $1.125, P=0.039$; OR $=1.060,95 \%$ CI: $1.001-1.123, P=$ 0.062 ; respectively) (Table 1). Meanwhile, There showed a causal effect of adiponectin on HOMA- $\beta$ and HOMA-IR by the IVW method $(\mathrm{OR}=1.048,95 \% \mathrm{CI}: 1.008-1.091, P=$ $0.020 ; \mathrm{OR}=1.089,95 \% \mathrm{CI}: 1.030-1.152, P=0.003$; respectively) and no causal effect existed among adiponectin and T2DM and FG (Table 1). After remove the ten pleiotropic IVs, there showed no causal effect among adiponectin and T2DM, HOMA- $\beta$, HOMA-IR, FG, whereas the results of IVW method showed a causal effect of adiponectin on FI $(0 \mathrm{R}=$ 1.057; 95\% CI: 1.004, 1.112; $P=0.034$ ) (Table 1).

\section{Heterogeneity and Sensitivity Tests}

Before removed the ten pleiotropic SNPs, Cochran's Q test (IVW method) and MR Egger intercept revealed showed 


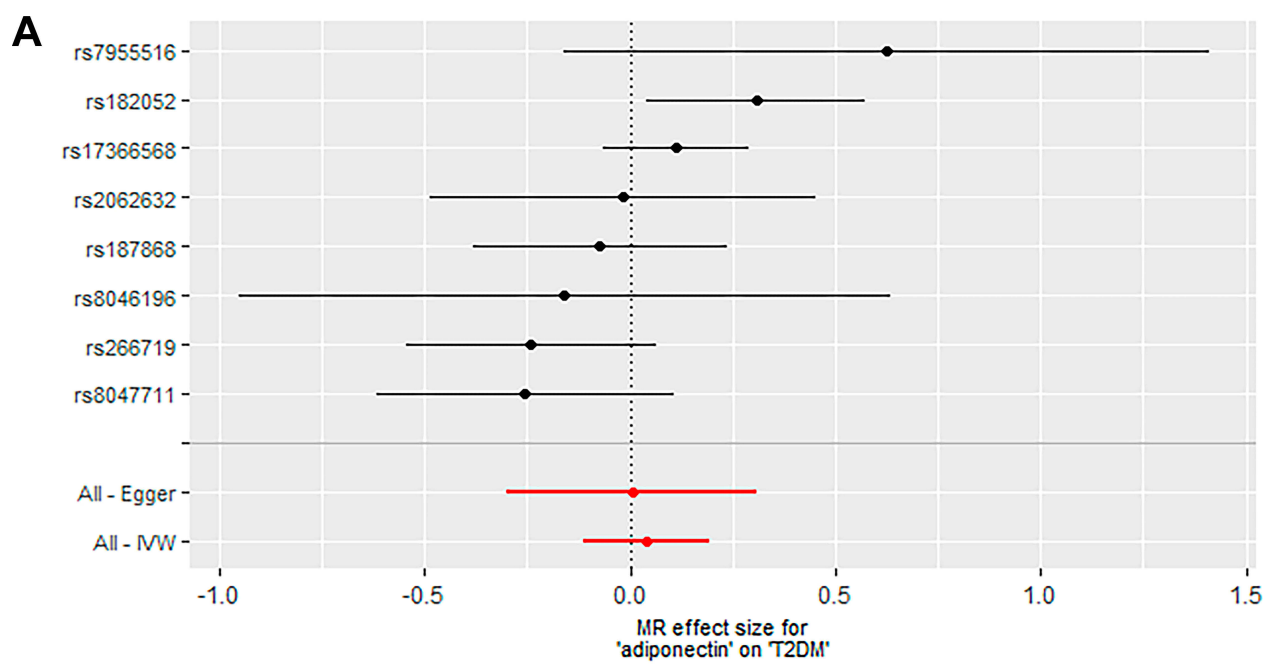

B

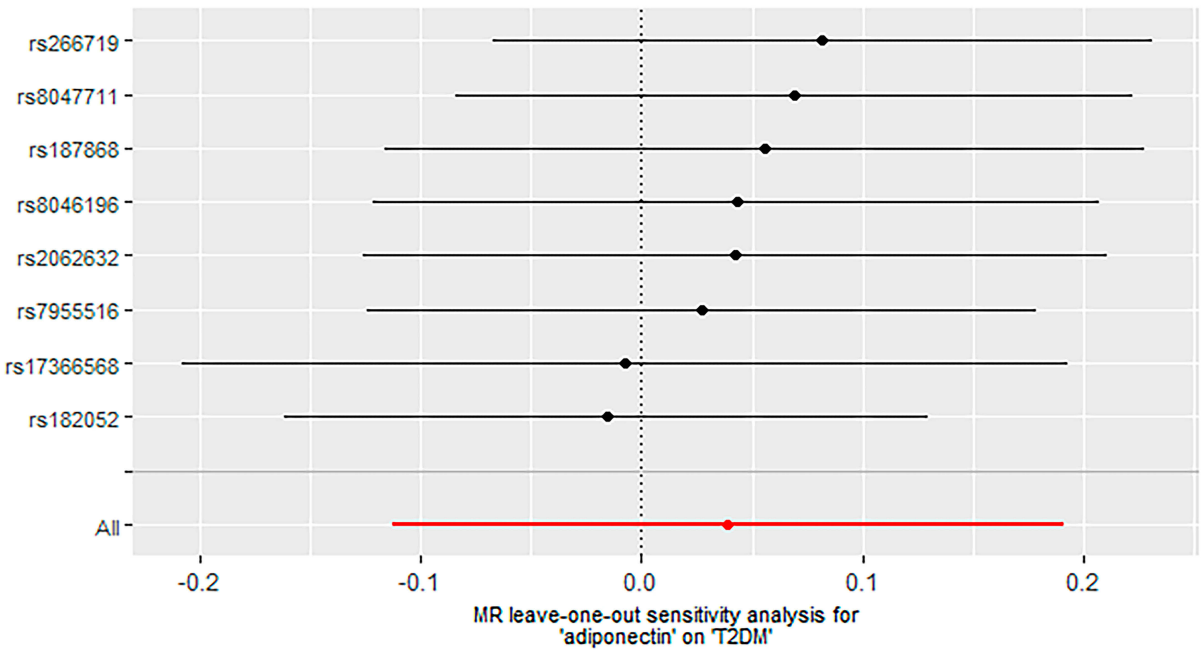

Figure 4 The forest plot of single SNP analysis and leave-one-out analysis depicting the relationship between adiponectin and T2DM with eight valid SNPs.

Notes: (A) The black point showed the causal effect estimate (beta coefficient) of adiponectin and T2DM utilizing a certain SNP, and the black line indicated the 95\% Cl of the estimate. The red point showed the causal effect estimate of adiponectin and T2DM with the eight valid SNPs using the Egger or IVW method, and the red lines indicated the $95 \% \mathrm{Cl}$ of the estimate. (B) Leave-one-out analysis depicted adiponectin-to-T2DM MR results (IVW method) by sequentially re-evaluating the causal estimate after discarding one IV at a time, which helped determine whether the overall effect was driven by one specific genetic variant. The black point was the causal effect estimate of adiponectin and T2DM after discarding a certain IV, and the black line indicated the $95 \% \mathrm{Cl}$ of estimate. The red point was the causal effect estimate of adiponectin and T2DM with the eight valid SNPs using the IVW methods, and the red line indicated the $95 \% \mathrm{Cl}$ of the estimate.

Abbreviations: SNPs, single-nucleotide polymorphisms; T2DM, type 2 diabetes mellitus; MR, Mendelian randomization; Egger, MR Egger regression; IVW, Inverse variance weighting; IV, instrumental variable; $\mathrm{Cl}$, confidence interval.

certain heterogeneity and directional pleiotropy existed among adiponectin and T2DM, HOMA-IR, FI, and no heterogeneity and directional pleiotropy existed among adiponectin and HOMA- $\beta$, FG (Table 2). After removed the ten pleiotropic SNPs, Cochran's Q test showed there were no heterogeneity $\left(P_{\text {MR-Egger }}=0.858, \quad P_{\mathrm{IVW}}=0.919 ; \quad P_{\text {MR-Egger }}>0.9\right.$ 99, $P_{\mathrm{IVW}}>0.999 ; \quad P_{\text {MR-Egger }}=0.998, P_{\mathrm{IVW}}=0.999 ;$ $P_{\text {MR-Egger }}=0.945, P_{\mathrm{IVW}}=0.943$; respectively) and directional pleiotropy (intercept $<-0.001, P=0.930$; intercept $=-0.001$, $P=0.803$; intercept $=-0.001, P=0.799$; intercept $=-0.0005$,
$P=0.875$, respectively) existed among adiponectin and HOMA- $\beta$, HOMA-IR, FI, FG, but showed the heterogeneity existed between adiponectin and T2DM $\left(P_{\text {MR-Egger }}=0.041\right.$; $\left.P_{\mathrm{IVW}}=0.065\right)($ Table 2). For T2DM, not all SNP analyses were meaningless, (eg, rs182052, Figure 4A), while "leaveone-out" analysis was symmetrical (Figure 4B); accordingly, excluding any SNP would not induce significant changes, namely, no obvious outliers existed. Singe SNP analyses were meaningless, that's, no outliers (Figure $5 \mathrm{~A}-\mathrm{C}$ ), and "leave-one-out" analysis was symmetrical for HOMA- $\beta$, 

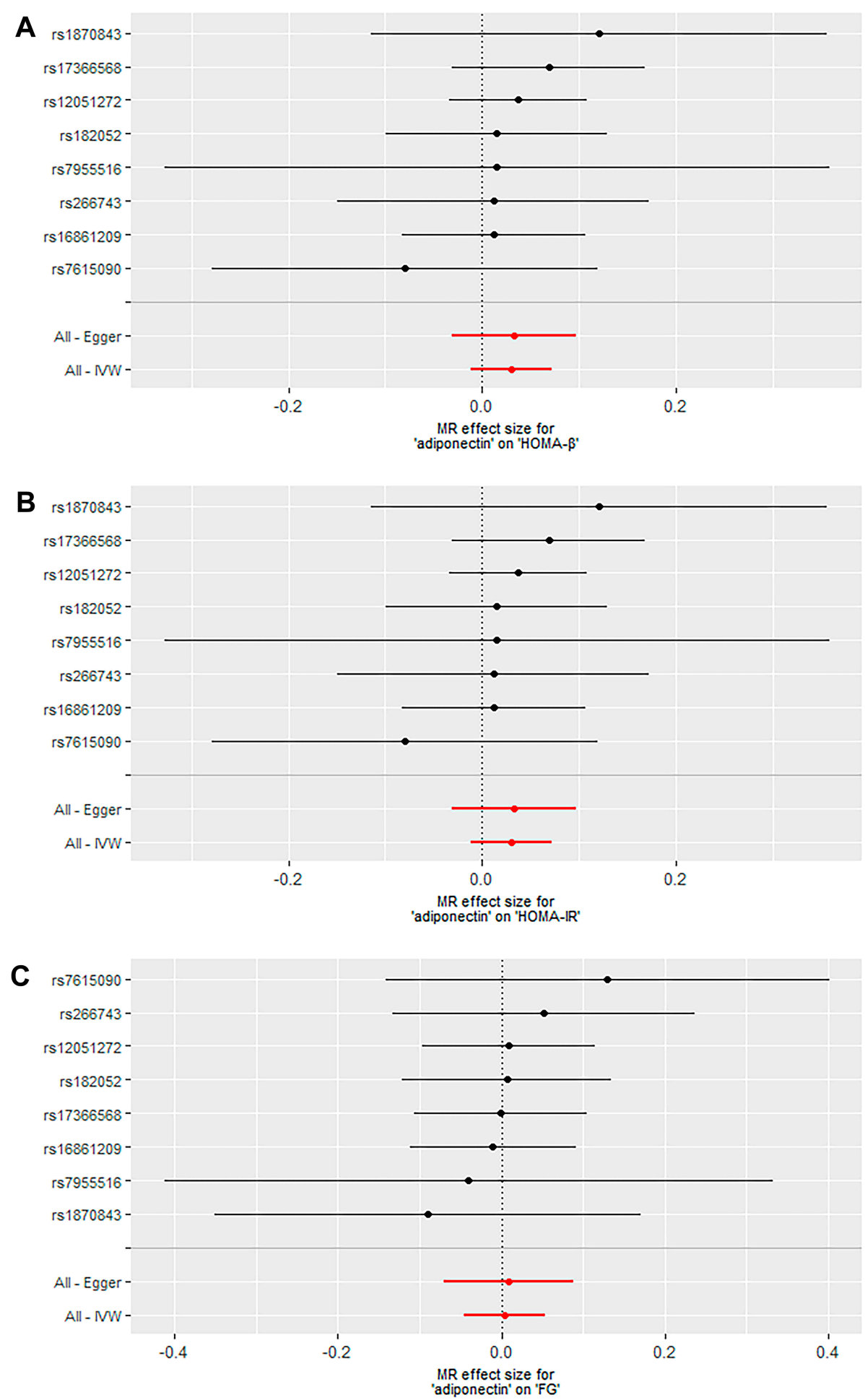

Figure 5 The forest plot of single SNP analysis depicting the relationship among adiponectin and HOMA- $\beta$, HOMA-IR, FG with eight valid SNPs.

Notes: (A) The black point showed the causal effect estimate (beta coefficient) of adiponectin and HOMA- $\beta$ utilizing a certain SNP, and the black line indicated the $95 \% \mathrm{Cl}$ of the estimate. The red point showed the causal effect estimate of adiponectin and HOMA- $\beta$ with the eight valid SNPs using the Egger or IVW method, and the red lines indicated the $95 \% \mathrm{Cl}$ of the estimate. (B) The black point showed the causal effect estimate (beta coefficient) of adiponectin and HOMA-IR utilizing a certain SNP, and the black line indicated the $95 \% \mathrm{Cl}$ of the estimate. The red point showed the causal effect estimate of adiponectin and HOMA-IR with the eight valid SNPs using the Egger or IVW method, and the red lines indicated the $95 \% \mathrm{Cl}$ of the estimate. (C) The black point showed the causal effect estimate (beta coefficient) of adiponectin and FG utilizing a certain SNP, and the black line indicated the $95 \% \mathrm{Cl}$ of the estimate. The red point showed the causal effect estimate of adiponectin and FG with the eight valid SNPs using the Egger or IVW method and the red lines indicated the $95 \% \mathrm{Cl}$ of the estimate.

Abbreviations: SNPs, single-nucleotide polymorphisms; Egger, MR Egger regression; IVW, Inverse variance weighting; HOMA-B, $\beta$-cell function index; HOMA-IR, insulin resistance; $\mathrm{FG}$, fasting glucose; $\mathrm{Cl}$, confidence interval. 
A

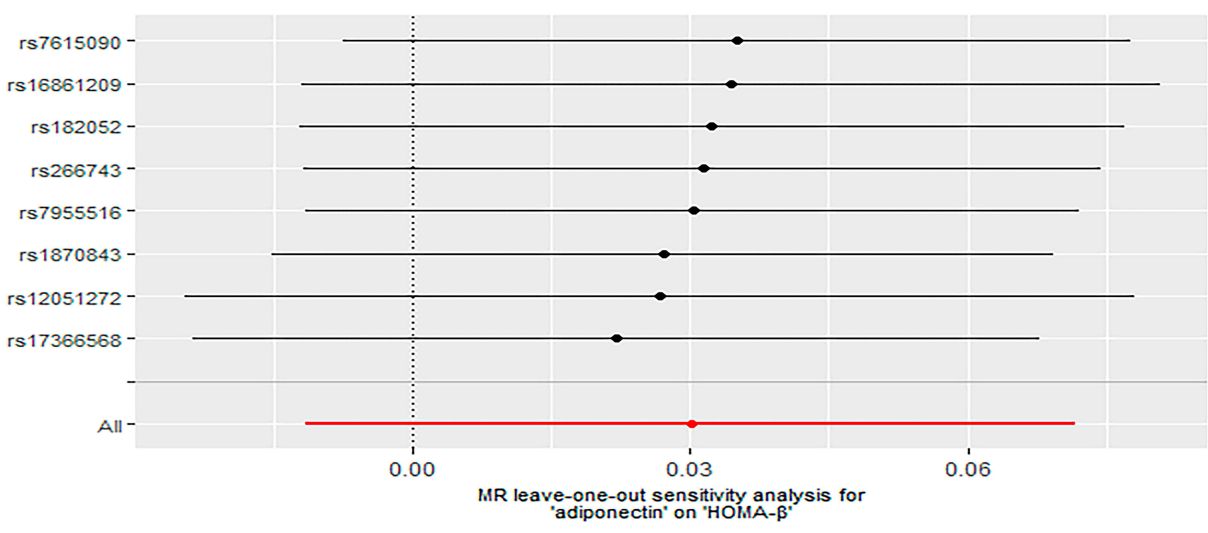

B

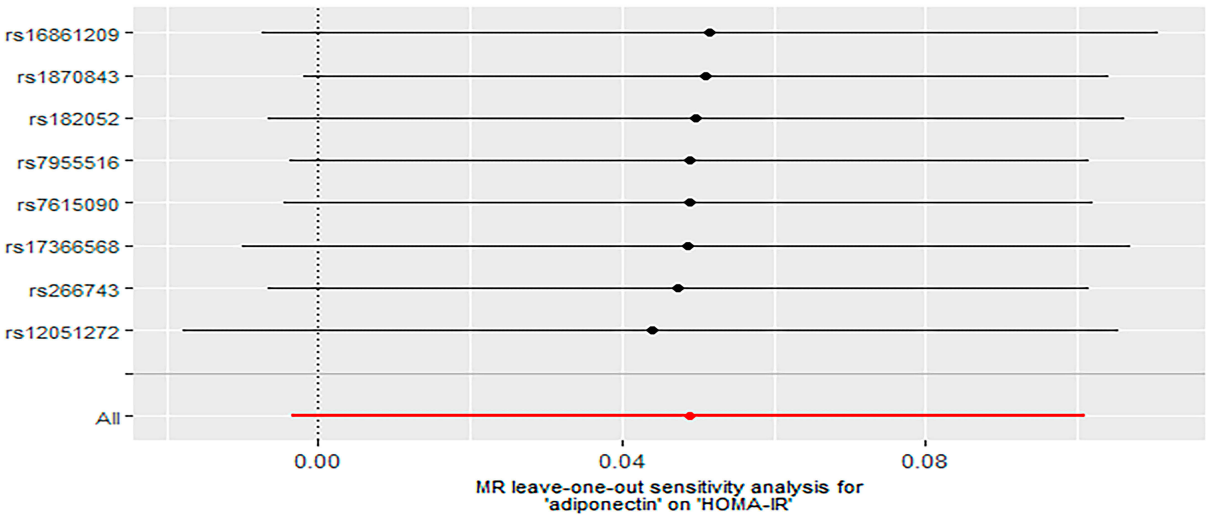

C

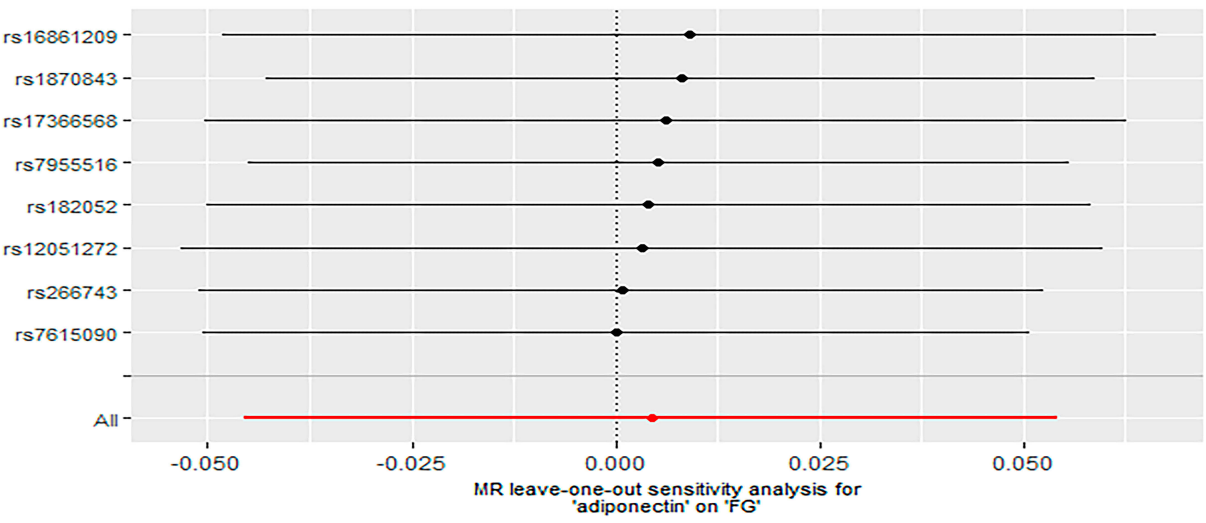

Figure 6 The forest plot of leave-one-out analysis depicting the relationship among adiponectin and HOMA- $\beta$, HOMA-IR, FG with eight valid SNPs.

Notes: Leave-one-out analysis depicted adiponectin-to-outcome MR results (IVW method) by sequentially re-evaluating the causal estimate after discarding one IV at a time, which helped determine whether the overall effect was driven by one specific genetic variant. (A) The black point was the causal effect estimate of adiponectin and HOMA- $\beta$ after discarding a certain IV, and the black line indicated the $95 \% \mathrm{Cl}$ of the estimate. The red point was the causal effect estimate of adiponectin and HOMA- $\beta$ with the eight valid SNPs using the IVW methods, and the red line indicated the $95 \% \mathrm{Cl}$ of the estimate. (B) The black point was the causal effect estimate of adiponectin and HOMA-IR after discarding a certain IV, and the black line indicated the $95 \% \mathrm{Cl}$ of estimate. The red point was the causal effect estimate of adiponectin and HOMA-IR with the eight valid SNPs using IVW methods, and the red line indicated the $95 \% \mathrm{Cl}$ of the estimate. (C) The black point was the causal effect estimate of adiponectin and FG after discarding a certain IV, and the black line indicated the $95 \% \mathrm{Cl}$ of estimate. The red point was the causal effect estimate of adiponectin and FG with the eight valid SNPs using IVW methods, and the red line indicated the $95 \% \mathrm{Cl}$ of the estimate.

Abbreviations: SNPs, single-nucleotide polymorphisms; Egger, MR Egger regression; IVW, Inverse variance weighting; HOMA-B, $\beta$-cell function index; HOMA-IR, insulin resistance; $\mathrm{FG}$, fasting glucose; $\mathrm{Cl}$, confidence interval.

HOMA-IR, and FG (Figure 6A-C, respectively), which were consistent with their sensitivity evaluation. For FI, four outliers were determined (rs17366568, rs1870843, rs7955516,
7615090; Figure 7A), and the "leave-one-out" analysis showed the IVW causal association estimate was driven by a single SNP (Figure 7B). 

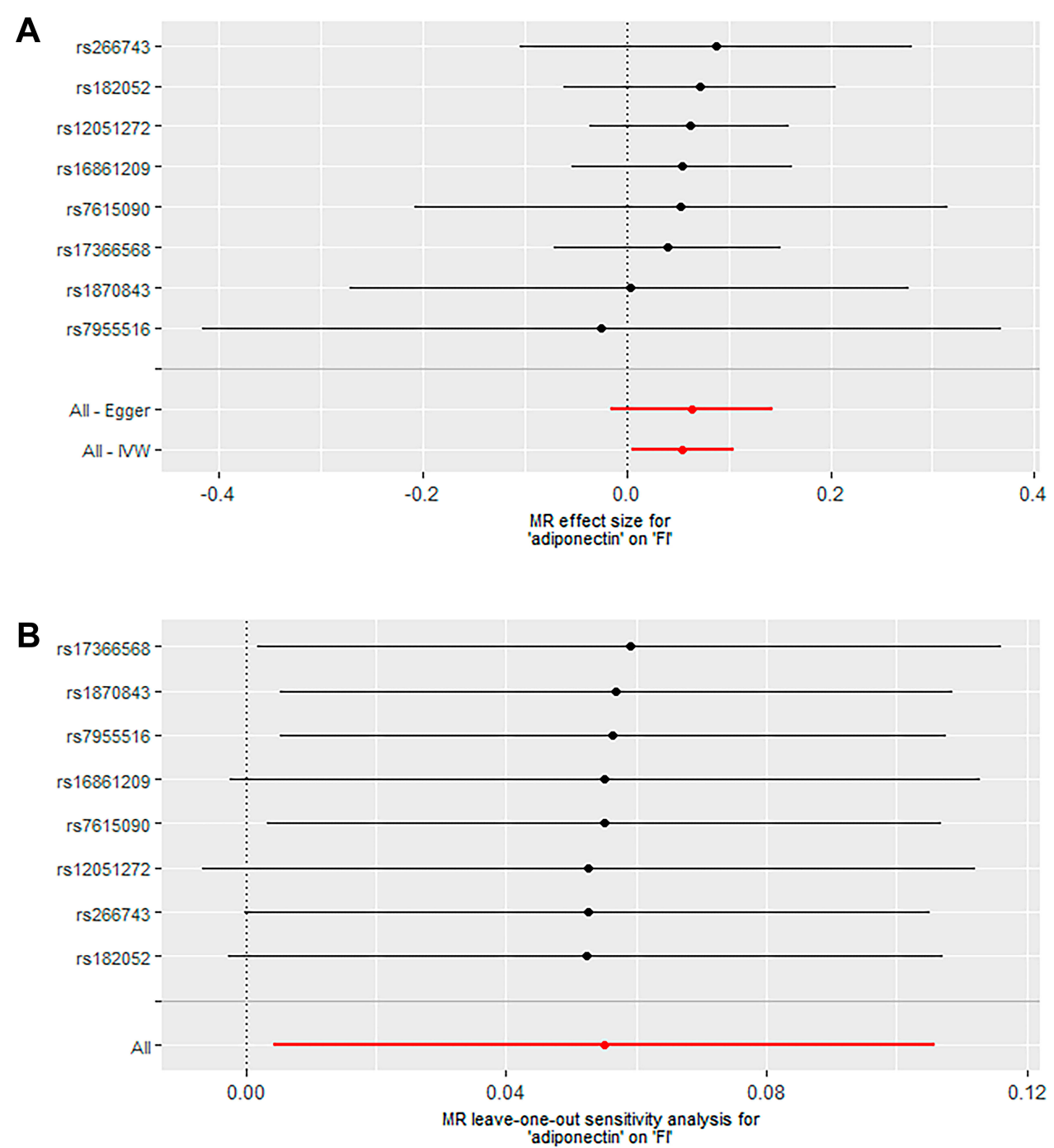

Figure 7 The forest plot of single SNP analysis and leave-one-out analysis depicting the relationship between adiponectin and FI with eight valid SNPs.

Notes: (A) The black point showed the causal effect estimate (beta coefficient) of adiponectin and $\mathrm{FI}$ utilizing a certain SNP, and the black line indicated the $95 \% \mathrm{Cl}$ of the estimate. The red point showed the causal effect estimate of adiponectin and FI with the eight valid SNPs using the Egger or IVW method, and the red lines indicated the $95 \%$ $\mathrm{Cl}$ of the estimate. (B) Leave-one-out analysis depicted adiponectin-to-FI MR results (IVW method) by sequentially re-evaluating the causal estimate after discarding one IV at a time, which helped determine whether the overall effect was driven by one specific genetic variant. The black point was the causal effect estimate of adiponectin and $\mathrm{FI}$ after discarding a certain IV, and the black line indicated the $95 \% \mathrm{Cl}$ of the estimate. The red point was the causal effect estimate of adiponectin and FI with the eight valid SNPs using the IVW methods, and the red line indicated the $95 \% \mathrm{Cl}$ of the estimate.

Abbreviations: SNPs, single-nucleotide polymorphisms; Egger, MR Egger regression; IVW, Inverse variance weighting; FI, fasting insulin; Cl, confidence interval.

\section{Discussion}

The relationship between adiponectin and HOMA-IR is controversial. People with extreme HOMA-IR have excessively high plasma adiponectin levels. Indeed, the inhibitory effect of hyperinsulinemia on plasma adiponectin occurred in females. ${ }^{45-48}$ Insulin infusion reduced plasma adiponectin in healthy volunteers ${ }^{49}$ but elevated in patients with type 1 diabetes mellitus. ${ }^{50}$ However, the hyperinsulinemia caused by HOMA-IR resulted in low plasma adiponectin levels rather than reverse using European summary data. ${ }^{51}$ Hence, an MR study regarding HOMA-
IR as the exposure and adiponectin as an outcome should be performed next.

In the present research, the association among adiponectin and HOMA- $\beta$, HOMA-IR, FI was causal before the elimination of pleiotropic SNPs; meanwhile, the Cochran's Q test and MR Egger regression revealed showed absolute heterogeneity and directional pleiotropy existed them. The results of five MR methods showed no causal effect, heterogeneity, and directional pleiotropy existed among adiponectin and HOMA- $\beta$, HOMA-IR, FI after the elimination of pleiotropic SNPs, suggesting that causal effect 


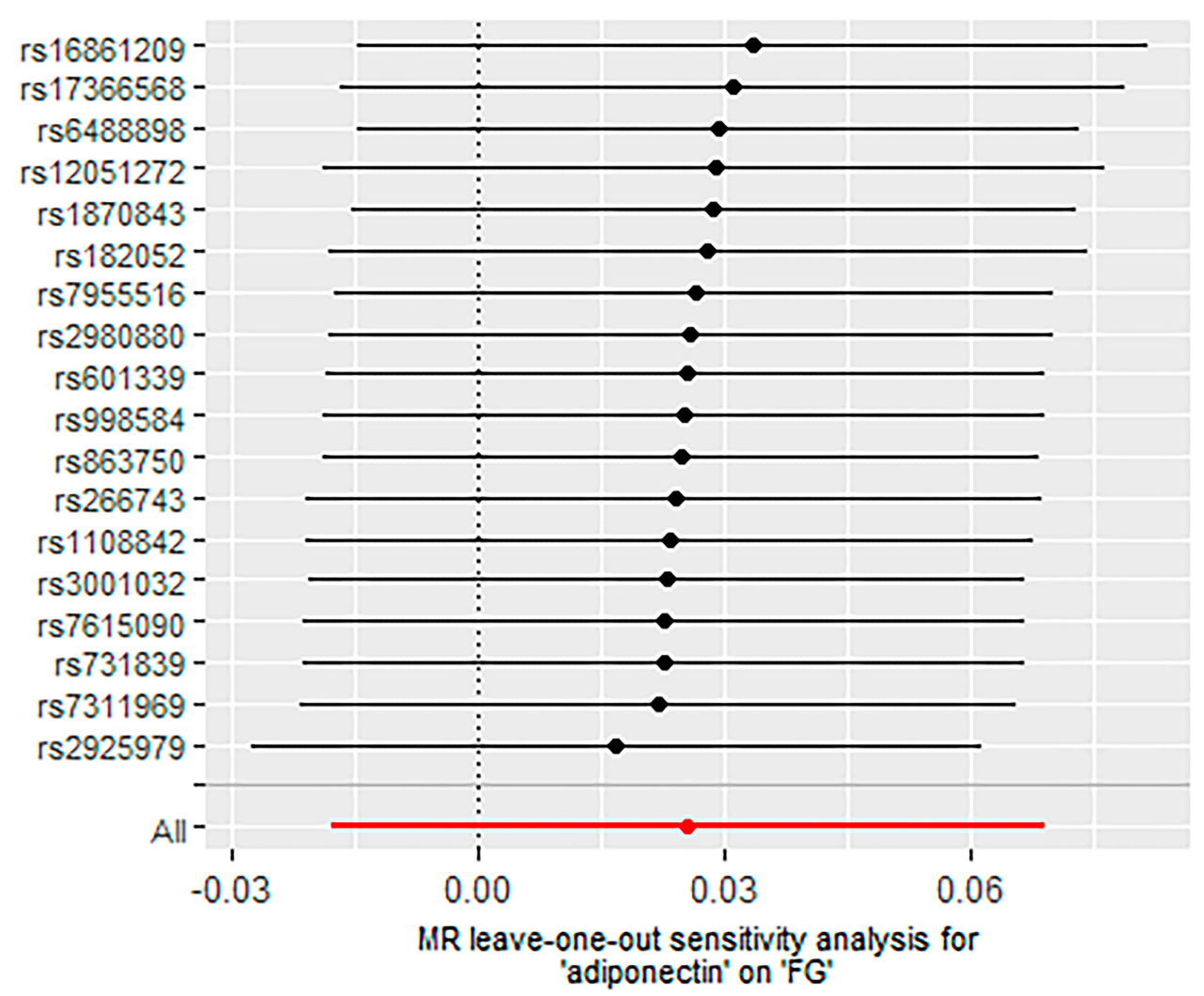

Figure 8 The forest plot of leave-one-out analysis depicting the relationship between adiponectin and FG without removing the pleiotropic IVs.

Notes: Leave-one-out analysis depicted adiponectin-to-FG MR results (IVW method) by sequentially re-evaluating the causal estimate after discarding one IV at a time, which helped determine whether the overall effect was driven by one specific genetic variant. The black point was the causal effect estimate of adiponectin and FG after discarding one IV, and the black line indicated the $95 \% \mathrm{Cl}$ of the estimate. The red point was the causal effect estimate of adiponectin and FG with the I8 valid SNPs using the IVW methods, and the red lines indicated the $95 \% \mathrm{Cl}$ of the estimate.

Abbreviations: SNPs, single-nucleotide polymorphisms; Egger, MR Egger regression; IVW, Inverse variance weighting; FG, fasting glucose; Cl, confidence interval.

before was likely mediated by pleiotropy. No causal effect, heterogeneity, and directional pleiotropy existed between adiponectin and FG before and after removing the pleiotropic SNPs, and leave-one-out analysis was symmetrical (Figure 8), which indicated obesity maybe is not the confounding factor. No causal effect existed between adiponectin and T2DM before and after removing the pleiotropic SNPs, but the heterogeneity still existed after removed the ten pleiotropic SNPs, which is more likely at the price of excessive proxies.

Our research did not provide evidence that decreasing circulating adiponectin levels, which were regulated by genetic factors, increased the risk of T2DM or glucose homeostasis. No causal effects were determined among adiponectin and T2DM, HOMA- $\beta$, HOMA-IR, FI with the power of $100 \%, 100 \%, 100 \%, 84 \%$, respectively (Table S5), which was consistent with the recent study. ${ }^{52}$ Any pharmaceutical and lifestyle interventions designed to alter adiponectin levels would not improve glucose homeostasis or prevent T2DM. ${ }^{51}$ The inconsistency of the estimates from different methods indicated that the genome-wide significant variants were not all valid IVs, and the causal conclusion based on these variants would be unreliable. ${ }^{53}$ Nevertheless, our results were consistent, and our IVs were valid. The findings suggested that circulating adiponectin concentration was more likely to be an epiphenomenon in the context of T2DM and glucose homeostasis than a key determinant, which was also consistent with the previous study. ${ }^{54}$

Our study exhibits several strengths. Firstly, we had larger samples than similar studies before. Secondly, we considered ethnicity into account, ie, the summary data of adiponectin, T2DM, and glucose homeostasis all came from the same ethnicity (European). The calculation of LD was also conducted on a special website using the European descent of 1000 Genomes Project. Furthermore, to the best of our knowledge, no existing MR analyses have investigated the causal relationship between adiponectin and HOMA- $\beta$, FG, FI. Our research is the first to investigate the causal relationship between them by the MR approach and a variety of sensitivity analyses. Lastly, the numbers of IVs 
were comprehensive, including liberal and conservative SNPs. Conservative SNPs were less likely to go against the MR assumption that the instrument should not affect the outcome other than the exposure of interest.

However, our research has its shortcomings. First, adiponectin exists as high-, medium-, and lowmolecular-weight isoforms in this research, namely total circulating adiponectin, which may have distinct functions. Second, we cannot account for complex feedback loops and rule out the possibility that the causalfree estimates among adiponectin and T2DM, glucose homeostasis were caused by age or sex. Although we did our best to manage the pleiotropic or confounding factors, the residual pleiotropic or confounding factors may remain. $^{27}$ Moreover, only eight SNPs of the 162 SNPs significantly associated with adiponectin were used as IVs, although limiting the numbers of IVs used in the MR study contributes to relieving the biases from weak instruments. Further, the MR analysis of adiponectin and T2DM was conducted with excessive proxies, which may produce unreliable results. ${ }^{55}$ Lastly, most IVs used in the MR research do not significantly associate with adiponectin in Asians, which is probable a lack of the related research, so performing further study on adiponectin in Asians is needed. And the subjects referred to in our study are European ancestry only, which helps minimize the likelihood of ethnicity bias, so performing MR analyses in other populations is expected in the future.

\section{Conclusion}

In all, our MR study revealed that adiponectin had no causal effect on T2DM and glucose homeostasis and the associations among them in observational studies may be due to confounding factors.

\section{Acknowledgments}

We express our gratitude to the ADIPOGen and MAGIC consortia and Dr.Angli Xue for providing the summary data. We are incredibly thankful to our teacher, who taught us about the Mendelian randomization, and our senior schoolmates with whom we learned considerably about mendelian randomization.

\section{Funding}

This study was funded by the National Natural Science Foundation of China (81770759), Major Project of Guangxi Innovation Driven (AA18118016), National Key
Research and Development Program of China (2017 YFC0908000).

\section{Disclosure}

The authors report no conflicts of interest in this work.

\section{References}

1. Miguel-Escalada I, Bonàs-Guarch S, Cebola I, et al. Human pancreatic islet three-dimensional chromatin architecture provides insights into the genetics of type 2 diabetes. Nat Genet. 2019;51 (7):1137-1148. doi:10.1038/s41588-019-0457-0

2. Long T, Wang J, Han X, et al. Association between resting heart rate and incident diabetes risk: a Mendelian randomization study. Acta Diabetol. 2019;56:1037-1044. doi:10.1007/s00592-019-01344-3

3. Zhou B, Lu Y, Hajifathalian K, et al. Worldwide trends in diabetes since 1980: a pooled analysis of 751 population-based studies with 4.4 million participants. Lancet (London, England). 2016;387 (10027):1513-1530. doi:10.1016/S0140-6736(16)00618-8

4. Xu Y, Wang L, He J, et al. Prevalence and control of diabetes in Chinese adults. JAMA. 2013;310(9):948-959. doi:10.1001/jama. 2013.168118

5. Li J, Ni J, Wu Y, et al. Sex differences in the prevalence, awareness, treatment, and control of diabetes mellitus among adults aged 45 years and older in rural areas of Northern China: a cross-sectional, population-based study. Front Endocrinol (Lausanne). 2019;10:147. doi:10.3389/fendo.2019.00147

6. Juma PA, Mohamed SF, Matanje Mwagomba BL, et al. Noncommunicable disease prevention policy process in five African countries authors. BMC Public Health. 2018;18(Suppl 1):961. doi:10.1186/s12889-018-5825-7

7. Younossi ZM, Golabi P, de Avila L, et al. The global epidemiology of NAFLD and NASH in Patients with type 2 diabetes: a systematic review and meta-analysis. J Hepatol. 2019;71:793-801. doi:10.1016/ j.jhep.2019.06.021

8. Zheng Y, Ley SH, Hu FB. Global aetiology and epidemiology of type 2 diabetes mellitus and its complications. Nat Rev Endocrinol. 2018;14(2):88-98. doi:10.1038/nrendo.2017.151

9. Willemsen G, Ward KJ, Bell CG, et al. The concordance and heritability of type 2 diabetes in 34,166 twin pairs from international twin registers: the discordant twin (DISCOTWIN) consortium. Twin Res Hum Genet. 2015;18(6):762-771. doi:10.1017/thg.2015.83

10. Dastani Z, Hivert MF, Timpson N, et al. Novel loci for adiponectin levels and their influence on type 2 diabetes and metabolic traits: a multi-ethnic meta-analysis of 45,891 individuals. PLoS Genet. 2012;8 (3): 1002607.

11. Yeung SLA, Schooling CM. Adiponectin and coronary artery disease risk: a bi-directional Mendelian randomization study. Int J Cardiol. 2018;268:222-226. doi:10.1016/j.ijcard.2018.03.132

12. Jee SH, Sull JW, Lee J-E, et al. Adiponectin concentrations: a genome-wide association study. Am J Hum Genet. 2010;87 (4):545-552. doi:10.1016/j.ajhg.2010.09.004

13. Wu Y, Li Y, Lange EM, et al. Genome-wide association study for adiponectin levels in Filipino women identifies CDH13 and a novel uncommon haplotype at KNG1-ADIPOQ. Hum Mol Genet. 2010;19 (24):4955-4964. doi: $10.1093 / \mathrm{hmg} / \mathrm{ddq} 423$

14. Chung $\mathrm{C}-\mathrm{M}$, Lin $\mathrm{T}-\mathrm{H}$, Chen $\mathrm{J}-\mathrm{W}$, et al. A genome-wide association study reveals a quantitative trait locus of adiponectin on $\mathrm{CDH} 13$ that predicts cardiometabolic outcomes. Diabetes. 2011;60(9):2417-2423. doi: $10.2337 / \mathrm{db} 10-1321$

15. Wu Y, Gao H, Li H, et al. A meta-analysis of genome-wide association studies for adiponectin levels in East Asians identifies a novel locus near WDR11-FGFR2. Hum Mol Genet. 2014;23(4):1108-1119. doi: $10.1093 / \mathrm{hmg} / \mathrm{ddt} 488$ 
16. Dastani Z, Johnson T, Kronenberg F, et al. The shared allelic architecture of adiponectin levels and coronary artery disease. Atherosclerosis. 2013;229(1):145-148. doi:10.1016/j.atherosclerosis. 2013.03.034

17. Yamauchi T, Nio Y, Maki T, et al. Targeted disruption of AdipoR1 and AdipoR2 causes abrogation of adiponectin binding and metabolic actions. Nat Med. 2007;13(3):332-339. doi:10.1038/nm1557

18. Borges MC, Oliveira IO, Freitas DF, et al. Obesity-induced hypoadiponectinaemia: the opposite influences of central and peripheral fat compartments. Int J Epidemiol. 2017;46(6):2044-2055. doi:10.1093/ ije/dyx 022

19. Zhuang H, Han J, Cheng L, Liu SL. A positive causal influence of IL-18 levels on the risk of T2DM: a mendelian randomization study. Front Genet. 2019;10:295. doi:10.3389/fgene.2019.00295

20. Swerdlow DI. Mendelian randomization and type 2 diabetes. Cardiovasc Drugs Ther. 2016;30(1):51-57. doi:10.1007/s10557016-6638-5

21. Dupuis J, Langenberg C, Prokopenko I, et al. New genetic loci implicated in fasting glucose homeostasis and their impact on type 2 diabetes risk. Nat Genet. 2010;42(2):105-116. doi:10.1038/ng.520

22. Weber KS, Strassburger K, Pacini G, et al. Circulating adiponectin concentration is inversely associated with glucose tolerance and insulin secretion in people with newly diagnosed diabetes. Diabet Med. 2017;34(2):239-244. doi:10.1111/dme.13278

23. Mente A, Meyre D, Lanktree MB, et al. Causal relationship between adiponectin and metabolic traits: a Mendelian randomization study in a multiethnic population. PLoS One. 2013;8(6):e66808. doi:10.1371/ journal.pone.0066808

24. Kashiwagi R, Yamada Y, Ito Y, et al. Increase in adiponectin level prevents the development of type 2 diabetes in Japanese men with low adiponectin levels. $J$ Endocr Soc. 2018;2(7):753-764 doi:10.1210/js.2018-00033

25. White J, Swerdlow DI, Preiss D, et al. Association of lipid fractions with risks for coronary artery disease and diabetes. JAMA Cardiol. 2016;1(6):692-699. doi:10.1001/jamacardio.2016.1884

26. Sattar N, Preiss D, Murray HM, et al. Statins and risk of incident diabetes: a collaborative meta-analysis of randomised statin trials. Lancet (London, England). 2010;375(9716):735-742. doi:10.1016/ S0140-6736(09)61965-6

27. Fall T, Xie W, Poon W, et al. Using genetic variants to assess the relationship between circulating lipids and type 2 diabetes. Diabetes. 2015;64(7):2676-2684. doi:10.2337/db14-1710

28. Warren LL, Li L, Nelson MR, et al. Deep resequencing unveils genetic architecture of ADIPOQ and identifies a novel low-frequency variant strongly associated with adiponectin variation. Diabetes. 2012;61(5):1297-1301. doi:10.2337/db11-0985

29. Bowden J, Davey Smith G, Haycock PC, Burgess S. Consistent estimation in mendelian randomization with some invalid instruments using a weighted median estimator. Genet Epidemiol. 2016;40 (4):304-314. doi:10.1002/gepi.21965

30. Hemani G, Zheng J, Elsworth B, et al. The MR-Base platform supports systematic causal inference across the human phenome. eLife. 2018;7. doi:10.7554/eLife.34408

31. Ortega Moreno L, Copetti M, Fontana A, et al. Evidence of a causal relationship between high serum adiponectin levels and increased cardiovascular mortality rate in patients with type 2 diabetes. Cardiovasc Diabetol. 2016;15:17. doi:10.1186/s12933-016-0339-z

32. Hartwig FP, Davies NM, Hemani G, Davey Smith G. Two-sample Mendelian randomization: avoiding the downsides of a powerful, widely applicable but potentially fallible technique. Int J Epidemiol. 2016;45(6):1717-1726. doi:10.1093/ije/dyx028

33. Ahmad OS, Morris JA, Mujammami M, et al. A Mendelian randomization study of the effect of type-2 diabetes on coronary heart disease. Nat Commun. 2015;6:7060. doi:10.1038/ncomms8060
34. Venkatrao M, Nagarathna R, Patil SS, et al. A composite of BMI and waist circumference may be a better obesity metric in Indians with high risk for type 2 diabetes: an analysis of NMB-2017, a nationwide cross-sectional study. Diabetes Res Clin Pract. 2020;161:108037. doi:10.1016/j.diabres.2020.108037

35. Bais S, Patel NJ. Role of adiponectin and its target receptors to control deposition of fat in obesity related disorders. $J$ Obes Med. 2019;16.

36. Lee S, Lacy ME, Jankowich M, Correa A, Wu W-C. Association between obesity phenotypes of insulin resistance and risk of type 2 diabetes in African Americans: the Jackson Heart Study. J Clin Transl Endocrinol. 2019;19:100210. doi:10.1016/j.jcte.2019.100210

37. Elizalde-Barrera CI, Rubio-Guerra AF, Lozano-Nuevo JJ, OlveraGomez JL. Triglycerides and waist to height ratio are more accurate than visceral adiposity and body adiposity index to predict impaired fasting glucose. Diabetes Res Clin Pract. 2019;153:49-54. doi:10.1016/j.diabres.2019.05.019

38. Davidsson L, Alkhabbaz E, Vijayan V, et al. Intermediate hyperglycaemia, insulin resistance and metabolic syndrome among obese Arab children (12-17 years old) in Kuwait. Prim Care Diabetes. 2020. doi:10.1016/j.pcd.2020.02.001

39. Xue A, Wu Y, Zhu Z, et al. Genome-wide association analyses identify 143 risk variants and putative regulatory mechanisms for type 2 diabetes. Nat Commun. 2018;9(1):2941. doi:10.1038/s41467018-04951-w

40. Shim H, Chasman DI, Smith JD, et al. A multivariate genome-wide association analysis of $10 \mathrm{LDL}$ subfractions, and their response to statin treatment, in 1868 Caucasians. PLoS One. 2015;10(4): e0120758. doi:10.1371/journal.pone.0120758

41. Hartwig FP, Davey Smith G, Bowden J. Robust inference in summary data Mendelian randomization via the zero modal pleiotropy assumption. Int J Epidemiol. 2017;46(6):1985-1998. doi:10.1093/ije/dyx102

42. Bowden J, Del Greco MF, Minelli C, et al. A framework for the investigation of pleiotropy in two-sample summary data Mendelian randomization. Stat Med. 2017;36(11):1783-1802. doi:10.1002/sim.7221

43. Bowden J, Davey Smith G, Burgess S. Mendelian randomization with invalid instruments: effect estimation and bias detection through Egger regression. Int J Epidemiol. 2015;44(2):512-525. doi:10.1093/ ije/dyv080

44. Brion MJ, Shakhbazov K, Visscher PM. Calculating statistical power in Mendelian randomization studies. Int $J$ Epidemiol. 2013;42 (5):1497-1501. doi:10.1093/ije/dyt179

45. Liu G, Ding M, Chiuve SE, et al. Plasma levels of fatty acid-binding protein 4 , retinol-binding protein 4 , high-molecular-weight adiponectin, and cardiovascular mortality among men with type 2 diabetes: a 22-year prospective study. Arterioscler Thromb Vasc Biol. 2016;36 (11):2259-2267. doi:10.1161/ATVBAHA.116.308320

46. Antuna-Puente B, Boutet E, Vigouroux C, et al. Higher adiponectin levels in patients with Berardinelli-Seip congenital lipodystrophy due to seipin as compared with 1-acylglycerol-3-phosphate-o-acyltransferase-2 deficiency. J Clin Endocrinol Metab. 2010;95(3):1463-1468. doi:10.1210/jc.2009-1824

47. Consitt LA, Saxena G, Schaefer M. Sex-dependent reductions in high molecular weight adiponectin during acute hyperinsulinemia are prevented with endurance training in older females. Clin Endocrinol (Oxf). 2018;88(5):673-682. doi:10.1111/cen.13563

48. Lee JY, Yang JW, Han BG, Choi SO, Kim JS. Adiponectin for the treatment of diabetic nephropathy. Korean J Intern Med. 2019;34 (3):480-491. doi:10.3904/kjim.2019.109

49. Basu R, Pajvani UB, Rizza RA, Scherer PE. Selective downregulation of the high molecular weight form of adiponectin in hyperinsulinemia and in type 2 diabetes: differential regulation from nondiabetic subjects. Diabetes. 2007;56(8):2174-2177. doi:10.2337/ db07-0185 
50. Pereira RI, Snell-Bergeon JK, Erickson C, et al. Adiponectin dysregulation and insulin resistance in type 1 diabetes. J Clin Endocrinol Metab. 2012;97(4):E642-E647. doi:10.1210/jc.2011-2542

51. Yaghootkar H, Lamina C, Scott RAet al,. Mendelian randomization studies do not support a causal role for reduced circulating adiponectin levels in insulin resistance and type 2 diabetes. Diabetes. 2013;62 (10):3589-3598.

52. Yaghootkar H, Lamina C, Scott RA, et al. Mendelian randomization studies do not support a causal role for reduced circulating adiponectin levels in insulin resistance and type 2 diabetes. Diabetes. 2013;62 (10):3589-3598. doi:10.2337/db13-0128

53. Burgess S, Bowden J, Fall T, Ingelsson E, Thompson SG. Sensitivity analyses for robust causal inference from mendelian randomization analyses with multiple genetic variants. Epidemiology. 2017;28 (1):30-42. doi:10.1097/EDE.0000000000000559
54. Borges MC, Barros AJD, Ferreira DLS, et al. Metabolic profiling of adiponectin levels in adults: mendelian randomization analysis. Circ Cardiovasc Genet. 2017;10:6. doi:10.1161/CIRCGENETICS.117. 001837

55. Luo Q, Wen Z, Li Y, et al. Assessment causality in associations between serum uric acid and risk of schizophrenia: a two-sample bidirectional mendelian randomization study. Clin Epidemiol. 2020;12:223-233.

\section{Publish your work in this journal}

Diabetes, Metabolic Syndrome and Obesity: Targets and Therapy is an international, peer-reviewed open-access journal committed to the rapid publication of the latest laboratory and clinical findings in the fields of diabetes, metabolic syndrome and obesity research. Original research, review, case reports, hypothesis formation, expert opinion and commentaries are all considered for publication. The manuscript management system is completely online and includes a very quick and fair peer-review system, which is all easy to use. Visit http://www.dovepress.com/testimonials.php to read real quotes from published authors. 